\title{
Analysis of the Educational Value of YouTube Laparoscopic Appendectomy Videos
}

\author{
Ki Bum Park, M.D. ${ }^{1}$, Moon Jin Kim, M.D. ${ }^{2}$, Jun Suh Lee, M.D., Ph.D. ${ }^{3}$ \\ 'Department of Surgery, Seoul St. Mary's Hospital, College of Medicine, The Catholic University of Korea, Seoul, ${ }^{2}$ Department of Surgery, Incheon St. \\ Mary's Hospital, College of Medicine, The Catholic University of Korea, Incheon, ${ }^{3}$ Department of Surgery, Seoul National University Bundang Hospital, Seoul \\ National University College of Medicine, Seongnam, Korea
}

Purpose: To evaluate the educational value of laparoscopic appendectomy (LA) videos on YouTube for surgical trainees.

Methods: The search term "Laparoscopic appendectomy" was used on YouTube. The top 100 videos sorted by the number of views were evaluated. Each YouTube account was analyzed, and only videos uploaded by medical physicians were included in this study. Video quality was evaluated using an arbitrary appendectomy scoring system. Video characteristics and Global Operative Assessment of Laparoscopic Skills (GOALS) scores were analyzed regarding video quality and upload source.

Results: The video quality of $14(25.0 \%)$ videos was graded as good, $36(64.3 \%)$ moderate, and 6 $(10.7 \%)$ of poor quality. Video characteristic analysis showed no differences in video quality according to the upload source ( $p=0.573$ ). Video quality and upload source were not related to video length, total views, days online, number of likes, number of dislikes, number of comments, or GOALS score. Among the factors analyzed, only appendicitis severity was found to be associated with video grade $(p=0.049)$.

Conclusion: The quality of LA YouTube videos varied. Categories considered as viewer feedback were not associated with video grade or upload source. Responsible video uploading by academic institutions, and appropriate censorship by YouTube seems necessary. Further research with objective data on actual application to surgical trainees is necessary.

Keywords: YouTube, Laparoscopy, Appendectomy, Online learning, Surgical training

This is an Open Access article distributed under the terms of the Creative Commons Attribution Non-Commercial License (http:// creativecommons.org/licenses/by-nc/4.0/) which permits unrestricted non-commercial use, distribution, and reproduction in any medium, provided the original work is properly cited.
Received June 19, 2019

Revised July 11, 2019

Accepted July 12, 2019

Corresponding author

Jun Suh Lee

Department of Surgery, Seoul

National University Bundang

Hospital, Seoul National University

College of Medicine, 82 Gumi-ro

173-gil, Bundang-gu, Seongnam

13620, Korea

Tel: +82-31-787-7111

Fax: +82-31-787-4078

E-mail: rudestock@gmail.com

ORCID:

https://orcid.org/0000-0001-9487-9826

\section{INTRODUCTION}

Internet access has become easier with the development of mobile devices. This has led to significant changes in the field of surgical education. ${ }^{1}$ One of these changes includes the use of medical information through video material, which is a form of multimedia learning. Education using multimedia has the advantage of converting cognitive input into long-term memory. ${ }^{2}$ According to a study by Rapp et al. ${ }^{3} 95 \%$ of surgical trainees have used video to prepare for surgery. In particular, 95\% reported obtaining information from YouTube. YouTube is world's largest free video sharing website, allowing users to freely upload, view, share, and comment on videos. ${ }^{4}$ Because of these features, YouTube is an important source for sharing medical information. However, the quality of medical information contents is not guaranteed because it is not a medical 
website.

Laparoscopic surgery has video-based features, and a large number of laparoscopic surgery videos have been uploaded on YouTube. Laparoscopic appendectomy (LA) is a surgical procedure primarily performed when surgical trainees begin to learn laparoscopic surgical techniques. ${ }^{5}$ There are many LA videos uploaded on YouTube, so it can be inferred that many, if not most, surgical trainees will access YouTube to view LA videos.

The authors of this study have previously analyzed YouTube laparoscopic cholecystectomy (LC) videos, and concluded that videos uploaded by tertiary centers were of better quality than those uploaded by secondary hospitals or private institutions. ${ }^{6}$ However, this quality discrepancy was not recognized by viewers. We sought to evaluate the quality of LA video content on YouTube, perform an analysis by video grade and upload source, and analyze viewer feedback.

\section{MATERIALS AND METHODS}

\section{Study design}

The term "laparoscopic appendectomy" was searched on YouTube, on May 1, 2018. The top 100 videos sorted by the number of views were evaluated. The "incognito mode" web browser was used so that search results are not affected by viewing history or cookies. Of the 100 videos, those uploaded by non-medical individuals via YouTube ID analysis were excluded. Animation or slide-based presentations, videos demonstrating other procedures such as an incisional hernia were excluded. Video characteristics including video length, total number of views, days online, views per day, likes, dislikes, number of comments, upload source, and appendicitis severity were reviewed. A Google search was performed for each YouTube ID to find the upload source. General hospitals and medical websites dedicated to medical education were classified as academic centers. Hospitals that were not general hospitals were classified as private centers. Videos with no hospital information were classified as independent surgeons. Appendicitis severity was classified as non-complicated appendicitis for normal looking or mildly inflamed appendix and complicated appendicitis for more severe cases, according to the appendicitis grading system proposed by Gomes et al. ${ }^{7}$

Institutional Review Board approval was unnecessary for this study, since only public access data was used.

\section{Evaluation of video quality}

Since there is no standardized system for evaluating the quality of the LA procedure, an arbitrary appendectomy procedure scoring system was devised by referring to textbooks and journals (Table 1). ${ }^{8-10}$ Each category was scored based on whether the entire procedure was illustrated in the video. A total score of $6 \sim 7$ points was classified as "good," 4 5 points as "moderate," and 3 points or less as "poor." The overall laparoscopic surgical technique was evaluated through Global Operative Assessment of Laparoscopic Skills (GOALS) score. ${ }^{11}$ The GOALS score is originally composed of 5 categories (depth perception, bimanual dexterity, efficiency, tissue handling, autonomy), and is graded on a 5-point Likert scale. The Autonomy category was excluded because it could not be determined through video analysis. Therefore, the maximum GOALS score was 20 points.

All researchers, who had more than 50 cases of LA experiences, assessed the quality of the video independently. Disagreements between the researchers about the scoring of a particular video were resolved by discussing the issue until a consensus was reached.

Table 1. Appendectomy procedure score

\begin{tabular}{|c|c|c|}
\hline Category & Assessment & Score \\
\hline \multirow[t]{2}{*}{ Inspection } & Not demonstrated & 0 \\
\hline & Inspection of entire abdomen & 1 \\
\hline \multirow[t]{2}{*}{ Mesoappendix ligation } & Not demonstrated & 0 \\
\hline & Demonstrated with proper hemostasis & 1 \\
\hline \multirow[t]{2}{*}{ Appendix ligation } & Stump length $>3 \mathrm{~mm}$ & 0 \\
\hline & Stump length $<3 \mathrm{~mm}$ & 1 \\
\hline \multirow{3}{*}{$\begin{array}{l}\text { Contamination material } \\
\text { spillage prevention }\end{array}$} & None & 0 \\
\hline & $\begin{array}{l}\text { Stump manipulation } \\
\text { (lumen electro-cauterization or endo- } \\
\text { stapler) }\end{array}$ & 1 \\
\hline & $\begin{array}{l}\text { Specimen manipulation } \\
\text { (grasper holding or endo-loop or } \\
\text { endo-stapler) }\end{array}$ & 1 \\
\hline \multirow[t]{2}{*}{ Appendix extraction } & Inappropriate & 0 \\
\hline & Use of the endo-bag & 1 \\
\hline \multirow[t]{2}{*}{ Peritoneal lavage } & Not demonstrated & 0 \\
\hline & $\begin{array}{l}\text { Irrigation } \\
\text { (Douglas pouch and } R L Q \text {, at least) }\end{array}$ & 1 \\
\hline Total score* & & $0 \sim 7$ \\
\hline
\end{tabular}




\section{Statistical analysis}

Data were analyzed using IBM SPSS statistics version 21.0 software (IBM Co., Armonk, NY, USA). Continuous variables were analyzed using Kruskal-Wallis analysis, with the Mann-Whitney test for Post-hoc comparison. The Bonferroni method was used to set the significance method. Categorical data were analyzed using Fisher's exact test. $p<0.05$ was considered significant, $p<0.017$ was considered significant in posthoc comparison.

\section{RESULTS}

Among the 100 videos identified, 44 were excluded. The excluded videos consisted of 3 videos uploaded by non-medical individuals, 16 non-surgical videos, 13 videos with poor quality for analysis, and 12 videos not demonstrating LA. After exclusion, a total of 56 videos were included in the study.

The quality of the videos was analyzed using the appendectomy procedure score. Overall, 14 (25\%) were graded as good, $36(64.3 \%)$ as moderate, and $6(10.7 \%)$ as poor. Table 2 shows the demographics of the videos by quality. The only characteristic that correlated with video grade was appendicitis severity ( $p=0.049)$. No difference was found in total views, days

Table 2. Analysis by video quality

\begin{tabular}{|c|c|c|c|c|c|}
\hline \multirow{2}{*}{ Video demographics } & \multicolumn{3}{|c|}{ Video quality } & \multirow{2}{*}{ Total } & \multirow{2}{*}{$p$ value } \\
\hline & Good & Moderate & Poor & & \\
\hline Videos & $14(25.0)$ & $36(64.3)$ & $6(10.7)$ & 56 & . \\
\hline Mean score & $6.36 \pm 0.50$ & $4.47 \pm 0.51$ & $2.33 \pm 1.03$ & $4.71 \pm 1.29$ & \\
\hline Inspection & $0.64 \pm 0.50$ & $0.19 \pm 0.40$ & $0.00 \pm 0.00$ & $0.29 \pm 0.46$ & 0.001 \\
\hline Mesoappendix ligation & $1.00 \pm 0.00$ & $0.94 \pm 0.23$ & $0.83 \pm 0.41$ & $0.95 \pm 0.23$ & 0.148 \\
\hline Stump ligation & $1.00 \pm 0.00$ & $0.92 \pm 0.28$ & $0.83 \pm 0.41$ & $0.93 \pm 0.26$ & 0.164 \\
\hline Specimen manipulation & $1.00 \pm 0.00$ & $0.97 \pm 0.17$ & $0.33 \pm 0.52$ & $0.91 \pm 0.29$ & $<0.001$ \\
\hline Stump manipulation & $0.93 \pm 0.27$ & $0.83 \pm 0.38$ & $0.00 \pm 0.00$ & $0.77 \pm 0.43$ & $<0.001$ \\
\hline Appendix extraction & $0.71 \pm 0.47$ & $0.39 \pm 0.49$ & $0.17 \pm 0.41$ & $0.45 \pm 0.50$ & 0.013 \\
\hline Peritoneal lavage & $0.79 \pm 0.43$ & $0.22 \pm 0.42$ & $0.17 \pm 0.41$ & $0.36 \pm 0.48$ & 0.001 \\
\hline Mean length & $11: 16 \pm 09: 39$ & $07: 39 \pm 06: 06$ & $05: 00 \pm 04: 40$ & $08: 16 \pm 07: 10$ & $0.028 *$ \\
\hline Mean views & $36,423 \pm 83,387$ & $28,995 \pm 52,962$ & $37,404 \pm 57,401$ & $31,753 \pm 61,172$ & 0.565 \\
\hline Days online & $1,784 \pm 884$ & $2,122 \pm 763$ & $2,171 \pm 1,074$ & $2,043 \pm 827$ & 0.490 \\
\hline Views per day & $34.1 \pm 87.7$ & $16.1 \pm 32.9$ & $35.3 \pm 74.9$ & $22.7 \pm 55.7$ & 0.966 \\
\hline Mean likes & $69.6 \pm 155.6$ & $81.6 \pm 309.7$ & $87.5 \pm 191.5$ & $79.2 \pm 264.8$ & 0.713 \\
\hline Mean dislikes & $6.9 \pm 15.4$ & $5.2 \pm 11.3$ & $6.8 \pm 13.3$ & $5.8 \pm 12.4$ & 0.872 \\
\hline Mean comments & $12.3 \pm 34.5$ & $20.8 \pm 77.7$ & $7.8 \pm 9.6$ & $16.0 \pm 62.7$ & 0.761 \\
\hline Upload source & & & & & 0.675 \\
\hline Independent surgeon & $6(42.9)$ & $17(47.2)$ & 2 (33.3) & 25 & \\
\hline Private center & 0 & $6(16.7)$ & 2 (33.3) & 8 & \\
\hline Academic center & $8(57.1)$ & $13(36.1)$ & 2 (33.3) & 23 & \\
\hline Appendicitis severity & & & & & 0.049 \\
\hline Non-complicated & $9(64.3)$ & 30 (83.3) & $6(100.0)$ & 45 & \\
\hline Complicated & $5(35.7)$ & $6(16.7)$ & 0 & 11 & \\
\hline GOALS & $18.93 \pm 1.27$ & $18.53 \pm 2.02$ & $17.83 \pm 2.40$ & $18.55 \pm 1.90$ & 0.625 \\
\hline
\end{tabular}


Table 3. Analysis by upload source

\begin{tabular}{|c|c|c|c|c|c|}
\hline \multirow{2}{*}{ Video demographics } & \multicolumn{3}{|c|}{ Video source } & \multirow{2}{*}{ Total } & \multirow{2}{*}{$p$ value } \\
\hline & Independent Surgeon & Private center & Academic center & & \\
\hline Videos & $25(44.6)$ & $8(14.3)$ & $23(41.1)$ & 56 & - \\
\hline Mean score & $4.88 \pm 1.13$ & $3.88 \pm 1.36$ & $4.83 \pm 1.37$ & $4.71 \pm 1.29$ & 0.216 \\
\hline Mean length & $09: 41 \pm 08: 28$ & $08: 04 \pm 06: 59$ & $06: 49 \pm 05: 31$ & $08: 16 \pm 07: 10$ & 0.198 \\
\hline Mean views & $19,911 \pm 29,493$ & $13,416 \pm 17,922$ & $51,002 \pm 87,379$ & $31,753 \pm 61,172$ & 0.351 \\
\hline Days online & $2,109 \pm 914$ & $2,150 \pm 695$ & $1,933 \pm 788$ & $2,043 \pm 827$ & 0.768 \\
\hline Views per day & $11.2 \pm 17.5$ & $5.8 \pm 7.5$ & $41.0 \pm 82.4$ & $22.7 \pm 55.7$ & 0.374 \\
\hline Mean likes & $27.8 \pm 44.1$ & $9.9 \pm 6.0$ & $159.1 \pm 402.1$ & $79.2 \pm 264.8$ & 0.143 \\
\hline Mean dislikes & $3.2 \pm 4.7$ & $1.9 \pm 1.8$ & $10.0 \pm 18.1$ & $5.8 \pm 12.4$ & 0.322 \\
\hline Mean comments & $8.3 \pm 17.1$ & $4.3 \pm 4.9$ & $28.3 \pm 96.0$ & $16.0 \pm 62.7$ & 0.661 \\
\hline Video quality & & & & & 0.573 \\
\hline Good & $6(24.0)$ & 0 & $8(34.8)$ & 14 & \\
\hline Moderate & $17(68.0)$ & $6(75.0)$ & $13(56.5)$ & 36 & \\
\hline Poor & $2(8.0)$ & $2(25.0)$ & $2(8.7)$ & 6 & \\
\hline Appendicitis severity & & & & & 0.222 \\
\hline Non-complicated & $22(88.0)$ & $6(75.0)$ & $17(73.9)$ & 45 & \\
\hline Complicated & $3(12.0)$ & $2(25.0)$ & $6(26.1)$ & 11 & \\
\hline GOALS & $18.56 \pm 1.61$ & $18.50 \pm 2.33$ & $18.57 \pm 2.11$ & $18.55 \pm 1.90$ & 0.863 \\
\hline
\end{tabular}

Values are presented as number $(\%)$ or mean \pm standard deviation.

online, likes, dislikes, number of comments, upload source, or GOALS score between the different video quality groups. Although the mean length differed based on the video quality $(p=0.028)$, the post-hoc test showed that the $\mathrm{p}$-value was higher than 0.017 , indicating that there was no significant difference.

Table 3 shows the demographics of videos by upload source. Overall, 25 (44.6\%) were independent surgeons, 8 (14.3\%) were private centers, and $23(41.1 \%)$ were academic centers. No statistically significant differences were seen in video quality, mean length, mean views, days online, views per day, mean likes, mean dislikes, mean number of comments, appendectomy severity, or GOALS score between the different sources of video uploaded.

\section{DISCUSSION}

Basic laparoscopic techniques are important in all fields of laparoscopic surgery. Inadequate techniques may cause potentially fatal complications. Therefore, it is essential for surgical trainees to learn these techniques properly at the beginning of their training. Surgical trainees most often begin their surgical training with LA, and YouTube is one of the most often sought training platforms. To our knowledge, this is the first study to examine the quality of YouTube videos of LA.

An important basic principle of surgery is to minimize postoperative complications. Although postoperative intra-abdominal abscess (IAA), such as stump appendicitis, has a low incidence, it is one of the most serious complications. ${ }^{12}$ The incidence of IAA depends on how well the stump and specimens are manipulated. The stump should be ligated to less than $3 \mathrm{~mm}$ to avoid stump appendicitis. ${ }^{8}$ In our analysis, this process had a high average score of 0.93 , suggesting that most surgeons in the videos included in this study were aware of the importance of this process. Visible mucosa must be electro-cauterized when the endo-loop is used in the stump ligation process. ${ }^{10}$ This is because a contaminated stump mucosa is likely to extrovert and there is risk of postoperative leak, which can lead to IAA. ${ }^{13}$ In this study, we found 13 videos not illustrating this process, including 10 without electro-cauterization; three were edited so it was unclear whether or not the procedure was performed. Since this procedure is a mandatory step that trainees must know, video content creators should not skip this process when editing. To prevent contaminated 
material dissemination of the specimen at the time of appendix division, the specimen opening must be held closed using a grasper or endo-loop. Videos received no points if the grasper or endo-loop was located too far from the opening or if the appendicolith or contaminated mucosa spread into the peritoneal cavity. However, most surgeons seemed to perform this procedure well, and the average score was 0.91 .

It is better to use an endo-bag when extracting the specimen from the abdominal cavity. ${ }^{9}$ Previous studies have suggested that directly extracting the specimen through the trocar is more cost-effective than specimen extraction using an endo-bag. ${ }^{14}$ However, from the videos observed in our study, during direct specimen extraction, even when the size of the specimen was very small, contaminated material was often observed in the trocar tip, which could potentially cause IAA or surgical site infection. Textbooks and the Society of American Gastroenterology Surgeons (SAGES) guideline recommend peritoneal lavage after specimen extraction, to reduce the incidence of IAA by washing contaminated materials with saline solution. ${ }^{9,10,15}$ However, some surgeons object to this guideline. St. Peter et al. ${ }^{16}$ reported that irrigation can increase the risk of IAA because it cannot reduce the microorganism load of bacteria attached to peritoneum mesothelial cells, is likely to spread contamination and can dilute the mediator of phagocytosis. In a recently published systemic review, the authors concluded that peritoneal lavage had no additional benefit and only lengthened operation time. ${ }^{17}$ In light of this controversy, we expect that trainees should first refer to textbooks and guidelines when learning LA theoretically, and include this procedure in the scoring table.

In principle, surgical videos for educational purpose should include a description of the patient position and trocar insertion site. However, most of the videos included in our study began with the insertion of the laparoscope. The reason for not including both steps is that it is not recorded with the laparoscope, and the process of recording this step before surgery or making animation is troublesome. We found one (4.3\%) video containing both steps from an academic center group; it seems that educators pay attention to these processes. Another procedure that requires more attention is inspection. As the video grade improved, the number of videos with inspection procedure increased. However, the average was 0.64 , even in the videos of good grade. Inspection is not a major procedure in the LA process but is an important opportunity to identify pathological findings not otherwise found in an imaging study.

In a recent systematic review, most studies frequently focused on whether the characteristics of YouTube videos were associated with the parameters video quality, upload source, and the number of likes. ${ }^{18}$ The authors of this study have previously analyzed LC YouTube videos and reported a qual- ity difference based on the upload source. ${ }^{6}$ Giovanni et al. ${ }^{19}$ also reported that there might be a quality difference based on the upload source in laparoscopic fundoplication YouTube videos. In our study, videos uploaded by academic centers had the highest percentage of good grade videos (57.1\%), but there were no statistically significant differences between the upload sources. This is likely because LA is a relatively easy procedure compared to previously analyzed procedures such as laparoscopic fundoplication or LC. Therefore, good quality videos can be recorded by centers of all sizes. The average of number of likes, although not statistically significant, was the highest in videos of poor grade. This is presumably because, as well as surgical trainees, many medical students and general public also watch a lot of videos to get information about LA. This suggests that the number of likes can be affected by the visual quality level of the video rather than demonstrating medical knowledge such as surgical procedure or technique in the video.

We classified appendicitis severity into two groups. The rate of complicated appendicitis was high in good grade videos. In complicated appendicitis cases, appendix inflammation is severe, and pericolic fluid due to perforation is likely to be present. The use of endo-staplers, endo-bags, and irrigation are often required in these cases, which may have led to higher scores. However, appendicitis severity is difficult to ascertain before actually viewing the video. Therefore, it is inappropriate to use it as a filtering condition for a good quality LA video.

YouTube allows the dissemination of health care information including information about surgery, but some videos are incorrect or misleading, according to a systemic review published in $2015 .^{20}$ In addition, most studies analyzing YouTube medical information seek to determine conditions that can filter good quality videos. It is not easy to identify a suitable condition to filter videos, because of the frequency with which they are uploaded. Video uploaders should always remember that numerous trainees will potentially watch their videos and thus, the authors should seek to improve the quality of video content through self-review before uploading, if peer review is difficult. Trainees watching LA YouTube videos should be aware that the quality of these videos is highly variable and that a high number of views or likes does not translate into an accurate or informative video.

We acknowledge that the study has some limitations. The most important limitation is the lack of actual application to surgical trainees. Further research including objective data after application, and trainees' subjective responses to these videos would contribute to this topic. The list of YouTube videos changes frequently, thus, the results of our study may differ if our study methods are used at a later date. Moreover, 
there is a possibility that when identifying the upload source, the YouTube ID may not correctly represent the uploader, since it can be created without any verification. Since there is no standardized scoring system to evaluate a LA procedure, an arbitrary scoring system was created and used. However, we expect that the observer-related bias is low because our evaluation method is not quantitative as in the 5-point Likert scale, but only determines whether each procedure is demonstrated in the videos.

In conclusion, the quality of LA YouTube videos varies. Categories that can be considered as viewer feedback were not associated with video grade or upload source. Multimedia learning using video content of questionable quality can be potentially dangerous. Responsible video uploading by academic institutions and appropriate censorship by YouTube are necessary. Further research with objective data on actual application to surgical trainees is necessary.

\section{ORCID}

Ki Bum Park, https://orcid.org/0000-0001-6035-6584

Moon Jin Kim, https://orcid.org/0000-0002-5404-3431

Jun Suh Lee, https://orcid.org/0000-0001-9487-9826

\section{AUTHORS' CONTRIBUTIONS}

Park KB and Lee JS designed the study, Park KB and Kim MJ performed data collection, Lee JS performed data analysis, and Park KB, Kim MJ and Lee JS wrote the manuscript.

\section{CONFLICT OF INTEREST}

None.

\section{FUNDING}

None.

\section{ACKNOWLEDGMENTS}

None.

\section{REFERENCES}

1) Pugh CM, Watson A, Bell RH, Jr., et al. Surgical education in the internet era. J Surg Res 2009;156:177-182.

2) Mayer RE. Applying the science of learning: evidence-based principles for the design of multimedia instruction. Am Psychol 2008;63:760-769.

3) Rapp AK, Healy MG, Charlton ME, Keith JN, Rosenbaum ME,
Kapadia MR. YouTube is the Most Frequently Used Educational Video Source for Surgical Preparation. J Surg Educ 2016;73:10721076.

4) Wikipedia contributors. YouTube [Internet]. Wikipedia, The Free Encyclopedia.; c2018 [cited on 2018 March 6]. Available from: https://en.wikipedia.org/wiki/YouTube.

5) Carrasco-Prats M, Soria Aledo V, Lujan-Mompean JA, RiosZambudio A, Perez-Flores D, Parrilla-Paricio P. Role of appendectomy in training for laparoscopic surgery. Surg Endosc 2003; 17:111-114.

6) Lee JS, Seo HS, Hong TH. YouTube as a potential training method for laparoscopic cholecystectomy. Ann Surg Treat Res 2015;89:92-97.

7) Gomes CA, Sartelli M, Di Saverio S, et al. Acute appendicitis: proposal of a new comprehensive grading system based on clinical, imaging and laparoscopic findings. World J Emerg Surg 2015;10:60.

8) Mangi AA, Berger DL. Stump appendicitis. Am Surg 2000;66:739741.

9) Richmond B. The Appendix. In: Townsend CM, Beauchamp RD, Evers BM, Mattox KL, editors. Sabiston textbook of surgery : the biological basis of modern surgical practice. 20th ed. Philadelphia, PA: Elsevier Saunders; 2017. p.1296-1311.

10) Thaler KJ, Miedema B. Laparoscopic surgery of the appendix and beyond-single incision and NOTES appendectomy. In: Swanström LL, Soper NJ, Leonard M, editors. Mastery of endoscopic and laparoscopic surgery. 4th ed. Philadelphia: Wolters Kluwer/Lippincott Williams \& Wilkins Health; 2014.

11) Vassiliou MC, Feldman LS, Andrew CG, et al. A global assessment tool for evaluation of intraoperative laparoscopic skills. Am J Surg 2005;190:107-113.

12) Martin LC, Puente I, Sosa JL, et al. Open versus laparoscopic appendectomy. A prospective randomized comparison. Ann Surg 1995;222:256-261; discussion 261-252.

13) Beldi G, Vorburger SA, Bruegger LE, Kocher T, Inderbitzin D, Candinas D. Analysis of stapling versus endoloops in appendiceal stump closure. Br J Surg 2006;93:1390-1393.

14) Gorter RR, Heij HA, Eker HH, Kazemier G. Laparoscopic appendectomy: State of the art. Tailored approach to the application of laparoscopic appendectomy? Best Pract Res Clin Gastroenterol 2014;28:211-224.

15) Korndorffer JR, Jr., Fellinger E, Reed W. SAGES guideline for laparoscopic appendectomy. Surg Endosc 2010;24:757-761.

16) St Peter SD, Holcomb GW, 3rd. Should peritoneal lavage be used with suction during laparoscopic appendectomy for perforated appendicitis? Adv Surg 2013;47:111-118.

17) Hajibandeh S, Hajibandeh S, Kelly A, et al. Irrigation Versus Suction Alone in Laparoscopic Appendectomy: Is Dilution the Solution to Pollution? A Systematic Review and Meta-Analysis. Surg Innov 2018;25:174-182. 
18) Drozd B, Couvillon E, Suarez A. Medical YouTube Videos and Methods of Evaluation: Literature Review. JMIR Med Educ 2018;4:e3.

19) Frongia G, Mehrabi A, Fonouni H, Rennert H, Golriz M, Gunther P. YouTube as a Potential Training Resource for Laparoscopic
Fundoplication. J Surg Educ 2016;73:1066-1071.

20) Madathil KC, Rivera-Rodriguez AJ, Greenstein JS, Gramopadhye AK. Healthcare information on YouTube: A systematic review. Health Informatics J 2015;21:173-194. 